\title{
Classification of Single Traveling Wave Solutions to the Generalized Strong Nonlinear Boussinesq Equation without Dissipation Terms in $P=1$
}

\author{
Xinghua Du \\ Department of Mathematics, Northeast Petroleum University, Daqing, China \\ Email: xinghuadu@126.com
}

Received January 15, 2014; revised February 15, 2014; accepted February 22, 2014

Copyright (c) 2014 Xinghua Du. This is an open access article distributed under the Creative Commons Attribution License, which permits unrestricted use, distribution, and reproduction in any medium, provided the original work is properly cited. In accordance of the Creative Commons Attribution License all Copyrights (C) 2014 are reserved for SCIRP and the owner of the intellectual property Xinghua Du. All Copyright (c) 2014 are guarded by law and by SCIRP as a guardian.

\section{ABSTRACT}

By the complete discrimination system for polynomial method, we obtained the classification of single traveling wave solutions to the generalized strong nonlinear Boussinesq equation without dissipation terms in $p=1$.

\section{KEYWORDS}

\section{Complete Discrimination System for Polynomial; Traveling Wave Solution; Generalized Strong Nonlinear Boussinesq Equation without Dissipation Terms}

\section{Introduction}

There are many methods of obtaining the exact solutions for nonlinear evolution equations, such as the homogeneous balance method [1], the inverse scattering method [2], Hirotas bilinear transformation [3], the extended tanh-function method [4], the sech-function method [5] and so on. Liu introduced complete discrimination system for the polynomial method to obtain the classification of traveling wave solutions to some nonlinear evolution equations [6-8]. In [9], the generalized strong nonlinear Boussinesq equation without dissipation terms was given by

$$
u_{t t}+\delta u_{x x x x}-\left(b_{1} u+b_{2} u^{p+1}+b_{3} u^{2 p+1}\right)_{x x}=0,
$$

where $\delta>0, p>0, b_{1}, b_{2}, b_{3}, p, \delta$ are constants. When $b_{3}=0, p=1$, Equation (1) becomes

$$
u_{t t}+\delta u_{x x x x}-\left(b_{1} u+b_{2} u^{2}\right)_{x x}=0,
$$

Equation (2) is an important model equation in physics. It describes the wave propagation in the weakly nonlinear and dispersive media. When $\delta>0, b_{1}=1$ or $\delta<0, b_{1}=1$, the Equation (2) becomes good Boussinesq equation $[10,11]$ or bad Boussinesq equation $[12,13]$. The good Boussinesq equation and bad Boussinesq equation have been studied by many authors [10-17]. But the classification of single traveling wave solutions to these equations hasn't been studied. In the present paper, we consider the following generalized strong nonlinear Boussinesq equation without dissipation terms in $p=1$ :

$$
u_{t t}+\delta u_{x x x x}-\left(b_{1} u+b_{2} u^{2}+b_{3} u^{3}\right)_{x x}=0,
$$

where $\delta>0, b_{1}, b_{2}, b_{3}, \delta$ are constants. By using Liu's method, the classification of single traveling wave solutions to Equation (3) is obtained. 


\section{The Traveling Wave Solutions to the Equation (3)}

Take wave transformation

$$
u(x, t)=u(\xi), \xi=k x-\omega t .
$$

Substituting Equation (4) into Equation (3) yields the following nonlinear ordinary difference equation:

$$
\omega^{2} u^{\prime \prime}+\delta k^{4} u^{(4)}-k^{2}\left(b_{1} u+b_{2} u^{2}+b_{3} u^{3}\right)^{\prime \prime}=0
$$

Integrating Equation (5) once with respect to $\xi$, and setting the integration constant to zero yields:

$$
\omega^{2} u^{\prime}+\delta k^{4} u^{\prime \prime \prime}-k^{2}\left(b_{1} u+b_{2} u^{2}+b_{3} u^{3}\right)^{\prime}=0 .
$$

Integrating Equation (6) twice with respect to $\xi$ yields:

$$
\left(u^{\prime}\right)^{2}=\frac{b_{3}}{2 \delta k^{2}} u^{4}+\frac{2 b_{2}}{3 \delta k^{2}} u^{3}+\frac{k^{2} b_{1}-\omega^{2}}{\delta k^{4}} u^{2}+c_{1} u+c_{0},
$$

where $c_{1}$ and $c_{0}$ are arbitrary constants.

In order to find the traveling wave solutions to the Equation (3), let us solve Equation (7). In this article, there are two cases to discuss the exact solutions of Equation (7) according to the arbitrary constant $c_{0}$.

Case 2.1 $c_{0}=0$, then Equation (7) becomes

$$
\left(u^{\prime}\right)^{2}=u\left(\frac{b_{3}}{2 \delta k^{2}} u^{3}+\frac{2 b_{2}}{3 \delta k^{2}} u^{2}+\frac{k^{2} b_{1}-\omega^{2}}{\delta k^{4}} u+c_{1}\right) .
$$

Integrating Equation (8) once yields

$$
\int \frac{\mathrm{d} u}{\sqrt{\varepsilon u F(u)}}= \pm \sqrt{\frac{\varepsilon b_{3}}{2 \delta k^{2}}}\left(\xi-\xi_{0}\right),
$$

where

$$
F(u)=u^{3}+\frac{4 b_{2}}{3 b_{3}} u^{2}+\frac{2\left(k^{2} b_{1}-\omega^{2}\right)}{k^{2} b_{3}} u+\frac{2 \delta k^{2} c_{1}}{b_{3}} .
$$

If $\frac{b_{3}}{\delta}>0$, we take $\varepsilon=1$; if $\frac{b_{3}}{\delta}<0$, we take $\varepsilon=-1$. The complete discrimination system for the third order polynomial $F(u)$ is given as follows:

$$
\begin{aligned}
& \Delta=\frac{\left(128 b_{2}^{3} k^{2}+1457 \delta k^{4} c_{1} b_{3}^{2}-648 b_{2} b_{3}\left(b_{1} k^{2}+\omega^{2}\right)\right)^{2}}{19683 b_{3}^{6} k^{4}}-\frac{4\left(18 k^{2} b_{1}-18 \omega^{2}-4 k^{3} b_{2}\right)^{3}}{729 k^{9} b_{3}^{3}}, \\
& D_{1}=\frac{162 k^{2} b_{1} b_{3}-162 b_{3} \omega^{2}-16 k^{2} b_{2}^{2}}{81 k^{2} b_{3}^{2}} .
\end{aligned}
$$

In order to obtain the solutions to the Equation (9), according to the complete discrimination system for the third order polynomial $F(u)$, there are four cases to be discussed.

Case 2.1.1. $\Delta=0, D_{1}<0 \quad F(u)=(u-\alpha)^{2}(u-\beta)$, where $\alpha, \beta$ are real constants, $\alpha \neq \beta$, $\beta>0$. If $\varepsilon=1$, when $\alpha>\beta$ and $u>\beta$, from Equation (9), we give the solution of Equation (7) as follows:

$$
\pm \sqrt{\frac{b_{3}}{2 \delta k^{2}}} \alpha(\alpha-\beta)\left(\xi-\xi_{0}\right)=\ln \frac{[\sqrt{\alpha(u-\beta)}-\sqrt{u(\alpha-\beta)}]^{2}}{|u-\beta|},
$$

when $\alpha<0$ and $u<\beta$, we have

$$
\pm \sqrt{\frac{b_{3}}{2 \delta k^{2}}} \alpha(\alpha-\beta)\left(\xi-\xi_{0}\right)=\ln \frac{[\sqrt{-\alpha(u-\beta)}-\sqrt{u(\beta-\alpha)}]^{2}}{|u-\beta|},
$$


when $\beta>\alpha>0$, we have

$$
\pm \sqrt{\frac{b_{3}}{2 \delta k^{2}}} \alpha(\beta-\alpha)\left(\xi-\xi_{0}\right)=\arcsin \frac{\alpha(u-\beta)+u(\alpha-\beta)}{|\beta(u-\alpha)|} .
$$

If $\varepsilon=-1$, when $\alpha>\beta$ and $u>\beta$, from Equation (9), we give the solutions of Equation (7)

$$
\pm \sqrt{-\frac{b_{3}}{2 \delta k^{2}}} \alpha(\beta-\alpha)\left(\xi-\xi_{0}\right)=\ln \frac{[\sqrt{\alpha(-u+\beta)}-\sqrt{u(\beta-\alpha)}]^{2}}{|u-\beta|},
$$

when $\alpha<0$, and $u<\beta$, we have

$$
\pm \sqrt{-\frac{b_{3}}{2 \delta k^{2}}} \alpha(\beta-\alpha)\left(\xi-\xi_{0}\right)=\ln \frac{[\sqrt{-\alpha(-u+\beta)}-\sqrt{u(\alpha-\beta)}]^{2}}{|u-\beta|},
$$

when $\beta>\alpha>0$, we have

$$
\pm \sqrt{-\frac{b_{3}}{2 \delta k^{2}}} \alpha(\alpha-\beta)\left(\xi-\xi_{0}\right)=\arcsin \frac{\alpha(-u+\beta)+u(\beta-\alpha)}{|\beta(u-\alpha)|} .
$$

Case 2.1.2. $\Delta=0, D_{1}=0 \quad F(u)=(u-\alpha)^{3}$, where $\alpha$ is real constant. If $\varepsilon=1$, when $u>\alpha$, we have

$$
u=\frac{2 \delta k^{2} \alpha}{\alpha^{2} b_{3}\left(\xi-\xi_{0}\right)^{2}-2 \delta k^{2}}+\alpha
$$

If $\varepsilon=-1$, when $u<\alpha$, we have

$$
u=\frac{2 \delta k^{2} \alpha}{-\alpha^{2} b_{3}\left(\xi-\xi_{0}\right)^{2}-2 \delta k^{2}}+\alpha
$$

Case 2.1.3. $\Delta>0, D_{1}<0 \quad F(u)=(u-\alpha)(u-\beta)(u-\gamma)$, where $\alpha, \beta, \gamma$ are different real constants. If $\varepsilon=1$, when $u<\gamma$, we have

$$
\begin{gathered}
u=\frac{-\gamma \alpha \operatorname{sn}^{2}\left(\sqrt{-\frac{\beta b_{3}}{2 \delta k^{2}}(\alpha-\beta)}\left(\xi-\xi_{0}\right), m\right)}{-\gamma \operatorname{sn}^{2}\left(\sqrt{-\frac{\beta b_{3}}{2 \delta k^{2}}(\alpha-\gamma)}\left(\xi-\xi_{0}\right), m\right)-(\alpha-\gamma)}, \\
u=\frac{-\gamma(\alpha-\beta) \operatorname{sn}^{2}\left(\sqrt{-\frac{\beta b_{3}}{2 \delta k^{2}}(\alpha-\beta)}\left(\xi-\xi_{0}\right), m\right)-\beta(\alpha-\gamma)}{-\gamma \operatorname{sn}^{2}\left(\sqrt{-\frac{\beta b_{3}}{2 \delta k^{2}}(\alpha-\gamma)}\left(\xi-\xi_{0}\right), m\right)-(\alpha-\gamma)},
\end{gathered}
$$

where $m^{2}=\frac{\gamma(\alpha-\beta)}{\beta(\alpha-\gamma)}$. If $\varepsilon=-1$, when $\gamma<u^{2}<\beta$, we have

$$
\begin{gathered}
u=\frac{-\beta \alpha \operatorname{sn}^{2}\left(\sqrt{-\frac{\beta b_{3}}{2 \delta k^{2}}(\alpha-\beta)}\left(\xi-\xi_{0}\right), m\right)+\alpha \beta}{-\alpha \operatorname{sn}^{2}\left(\sqrt{-\frac{\beta b_{3}}{2 \delta k^{2}}(\alpha-\gamma)}\left(\xi-\xi_{0}\right), m\right)+\beta}, \\
u=\frac{-\gamma \beta}{(\beta-\gamma) \operatorname{sn}^{2}\left(\sqrt{-\frac{\beta b_{3}}{2 \delta k^{2}}(\alpha-\gamma)}\left(\xi-\xi_{0}\right), m\right)-\beta},
\end{gathered}
$$


where $m^{2}=\frac{\alpha(\beta-\gamma)}{\beta(\alpha-\gamma)}$.

Case 2.1.4. $\Delta<0, F(u)=(u-\alpha)\left[(u-l)^{2}+s^{2}\right]$, where $\alpha, l, s$ are all real constants, and $\alpha>0, l, s>0$, we have

$$
u=\frac{a \operatorname{cn}\left(\frac{\sqrt{\mp \frac{s m_{1} \alpha b_{3}}{\delta k^{2}}}}{m m_{1}}\left(\xi-\xi_{0}\right), m\right)+b}{\operatorname{ccn}\left(\frac{\sqrt{\mp \frac{s m_{1} \alpha b_{3}}{\delta k^{2}}}}{m m_{1}}\left(\xi-\xi_{0}\right), m\right)+d},
$$

where

$$
a=\frac{1}{2} \alpha(c-d), b=\frac{1}{2} \alpha(d-c), c=\alpha-l-\frac{s}{m_{1}}, \quad d=-l-s m_{1}, E=\frac{s^{2}-l(\alpha-l)}{s \alpha}, m_{1}=E \pm \sqrt{E^{2}+1}, m^{2}=\frac{1}{1+m_{1}^{2}} .
$$

Case 2.2 $\quad c_{0} \neq 0 \quad$ In order to solve Equation (7), when $\frac{b_{3}}{\delta}>0$, we take the transformation as follows

$$
w=\left(\frac{b_{3}}{2 \delta k^{2}}\right)^{\frac{1}{4}}\left(u+\frac{b_{2}}{3 b_{3}}\right), \xi_{1}=\left(\frac{b_{3}}{2 \delta k^{2}}\right)^{\frac{1}{4}} \xi .
$$

Combining the expression (7) with Equation (25) yields

$$
w_{\xi_{1}}^{2}=F(w)=w^{4}+p w^{2}+q w+r,
$$

where

$$
\begin{gathered}
p=\frac{\sqrt{2}\left(k^{2} b_{1}-\omega^{2}\right)}{k^{3} \sqrt{\delta b_{3}}}, q=\frac{b_{2}^{3} k^{2}-18 k^{2} b_{1} b_{2} b_{3}+18 \omega^{2} b_{1} b_{2} b_{3}+27 \delta k^{4} b_{3}^{2}}{27 \delta k^{4} b_{3}^{2}}\left(\frac{b_{3}}{2 \delta k^{2}}\right)^{-\frac{1}{4}}, \\
\text { And } r=\frac{-b_{2}^{4} k^{2}+6 b_{2} b_{3} k^{2} b_{1}-6 b_{2} b_{3} \omega^{2}-18 c_{1} \delta k^{4} b_{2} b_{3}^{2}}{54 \delta k^{4} b_{3}^{3}}+c_{0} .
\end{gathered}
$$

When $\frac{b_{3}}{\delta}<0$, we take the following transformation:

$$
w=\left(-\frac{b_{3}}{2 \delta k^{2}}\right)^{\frac{1}{4}}\left(u+\frac{b_{2}}{3 b_{3}}\right), \xi_{1}=\left(-\frac{b_{3}}{2 \delta k^{2}}\right)^{\frac{1}{4}} \xi .
$$

Combining the expression (7) with Equation (27) yields

$$
w_{\xi_{1}}^{2}=-F(w)=-\left(w^{4}+p w^{2}+q w+r\right),
$$

where

$$
\begin{gathered}
p=-\frac{\sqrt{2}\left(k^{2} b_{1}-\omega^{2}\right)}{k^{3} \sqrt{-\delta b_{3}}}, q=\frac{b_{2}^{3} k^{2}-18 k^{2} b_{1} b_{2} b_{3}+18 \omega^{2} b_{1} b_{2} b_{3}+27 \delta k^{4} b_{3}^{2}}{-27 \delta k^{6} b_{3}^{2}}\left(-\frac{b_{3}}{2 \delta k^{2}}\right)^{-\frac{1}{4}}, \\
\text { and } r=\frac{b_{2}^{4} k^{2}-6 b_{2} b_{3} k^{2} b_{1}+6 b_{2} b_{3} \omega^{2}+18 c_{1} \delta k^{4} b_{2} b_{3}^{2}}{54 \delta k^{4} b_{3}^{3}}-c_{0} .
\end{gathered}
$$

The complete discrimination system for the fourth order polynomial $F(w)=w^{4}+p w^{2}+q w+r$ as follows: 


$$
\begin{aligned}
& D_{1}=4, D_{2}=-p, D_{3}=8 r p-2 p^{3}-9 q^{2}, \\
& D_{4}=4 p^{4} r-p^{3} q^{2}+36 p r q^{2}-32 r^{2} p^{2}-\frac{27}{4} q^{4}+64 r^{3}, E_{2}=9 p^{2}-32 p r .
\end{aligned}
$$

In order to obtain the solutions to Equation (26) and Equation (28), according to the complete discrimination system for the fourth order polynomial $F(w)$, there are nine cases to be discussed.

Case 2.2.1 $D_{2}<0, D_{3}=0$, and $D_{4}=0$, then $F(w)=\left((w-l)^{2}+s^{2}\right)^{2}$. where $s>0$. For $\frac{b_{3}}{\delta}>0$, the solution of Equation (7) is

$$
u=\left(\frac{b_{3}}{2 \delta k^{2}}\right)^{-\frac{1}{4}}\left[s \tan \left(s\left(\xi_{1}-\xi_{0}\right)\right)+l\right]-\frac{b_{2}}{3 b_{3}} .
$$

Case 2.2.2. $D_{2}=0, D_{3}=0$, and $D_{4}=0$, then $F(w)=w^{4}$. For $\frac{b_{3}}{\delta}>0$, the solution of Equation (7) is

$$
u=-\left(\frac{b_{3}}{2 \delta k^{2}}\right)^{-\frac{1}{2}}\left(\xi-\xi_{0}\right)^{-1}-\frac{b_{2}}{3 b_{3}} .
$$

Case 2.2.3. $D_{2}>0, D_{3}=0, \quad D_{4}=0$ and $E_{2}>0, \quad F(w)=(w-\alpha)^{2}(w-\beta)^{2}$, where $\alpha>\beta$. For $\frac{b_{3}}{\delta}>0$, when $w>\alpha$ or $w<\beta$, the solution of Equation (7) is

$$
u=\left(\frac{b_{3}}{2 \delta k^{2}}\right)^{-\frac{1}{4}}\left[\frac{\beta-\alpha}{2}\left[\operatorname{coth} \frac{\left(\frac{b_{3}}{2 \delta k^{2}}\right)^{\frac{1}{4}}(\alpha-\beta)\left(\xi-\xi_{0}\right)}{2}-1\right]+\beta\right]-\frac{b_{2}}{3 b_{3}},
$$

when $\beta<w<\alpha$, the solution of Equation (7) is

$$
u=\left(\frac{b_{3}}{2 \delta k^{2}}\right)^{-\frac{1}{4}}\left[\frac{\beta-\alpha}{2}\left[\tanh \frac{\left(\frac{b_{3}}{2 \delta k^{2}}\right)^{\frac{1}{4}}(\alpha-\beta)\left(\xi-\xi_{0}\right)}{2}-1\right]+\beta\right]-\frac{b_{2}}{3 b_{3}}
$$

Case 2.2.4. $D_{2}>0, D_{3}=0, \quad D_{4}=0$ and $E_{2}=0$, then $F(w)=(w-\alpha)^{3}(w-\beta)$, when $\frac{b_{3}}{\delta}>0$, $w>\alpha, w>\beta$ or $w<\alpha, w<\beta$, the solution of Equation (7) is

$$
u=\left(\frac{b_{3}}{2 \delta k^{2}}\right)^{-\frac{1}{4}}\left[\frac{4(\alpha-\beta)}{(\beta-\alpha)^{2}\left(\frac{b_{3}}{2 \delta k^{2}}\right)^{\frac{1}{2}}\left(\xi-\xi_{0}\right)^{2}-4}+\alpha\right]-\frac{b_{2}}{3 b_{3}},
$$

when $\frac{b_{3}}{\delta}<0, w>\alpha, w<\beta$ or $w<\alpha, w>\beta$, the solution of Equation (7) is

$$
u=\left(-\frac{b_{3}}{2 \delta k^{2}}\right)^{-\frac{1}{4}}\left[\frac{4(\alpha-\beta)}{-(\beta-\alpha)^{2}\left(-\frac{b_{3}}{2 \delta k^{2}}\right)^{\frac{1}{2}}\left(\xi-\xi_{0}\right)^{2}-4}+\alpha\right]-\frac{b_{2}}{3 b_{3}} .
$$


Case 2.2.5 $D_{2}>0, D_{3}>0$, and $D_{4}=0$, then $F(w)=(w-\alpha)^{2}(w-\beta)(w-\gamma)$. If $\frac{b_{3}}{\delta}>0$, when $\alpha>\beta$ and $w>\beta$ or when $\alpha<\gamma$ and $w<\gamma$, we have

$$
\begin{aligned}
& \exp \left\{ \pm\left(\frac{b_{3}}{2 \delta k^{2}}\right)^{\frac{1}{4}}(\alpha-\beta)(\alpha-\gamma)\left(\xi-\xi_{0}\right)\right\} \\
& =\frac{\left\{\sqrt{\left(\frac{b_{3}}{2 \delta k^{2}}\right)^{\frac{1}{4}}\left(u+\frac{b_{2}}{3 b_{3}}-\beta\right)(\alpha-\gamma)}-\sqrt{\left(\left(\frac{b_{3}}{2 \delta k^{2}}\right)^{\frac{1}{4}}\left(u+\frac{b_{2}}{3 b_{3}}\right)-\gamma\right)(\alpha-\beta)}\right\}^{2}}{\left|\left(\frac{b_{3}}{2 \delta k^{2}}\right)^{\frac{1}{4}}\left(u+\frac{b_{2}}{3 b_{3}}\right)-\alpha\right|},
\end{aligned}
$$

when $\alpha>\beta$ and $w<\gamma$, or when $\alpha<\gamma$, and $w<\beta$, we have

$$
\begin{aligned}
& \exp \left\{ \pm\left(\frac{b_{3}}{2 \delta k^{2}}\right)^{\frac{1}{4}}(\alpha-\beta)(\alpha-\gamma)\left(\xi-\xi_{0}\right)\right\} \\
& =\frac{\left\{\sqrt{\left(\left(\frac{b_{3}}{2 \delta k^{2}}\right)^{\frac{1}{4}}\left(u+\frac{b_{2}}{3 b_{3}}\right)-\beta\right)(\gamma-\alpha)}-\sqrt{\left(\left(\frac{b_{3}}{2 \delta k^{2}}\right)^{\frac{1}{4}}\left(u+\frac{b_{2}}{3 b_{3}}\right)-\gamma\right)(\beta-\alpha)}\right\}^{2}}{\left|\left(\frac{b_{3}}{2 \delta k^{2}}\right)^{\frac{1}{4}}\left(u+\frac{b_{2}}{3 b_{3}}\right)-\alpha\right|},
\end{aligned}
$$

when $\beta>\alpha>\gamma$, we have

$$
\begin{aligned}
& \pm \sin \left(\left(\frac{b_{3}}{2 \delta k^{2}}\right)^{\frac{1}{4}}(\beta-\alpha)(\alpha-\gamma)\left(\xi-\xi_{0}\right)\right) \\
& =\frac{\left[\left(\frac{b_{3}}{2 \delta k^{2}}\right)^{\frac{1}{4}}\left(u+\frac{b_{2}}{3 b_{3}}\right)-\beta\right](\alpha-\gamma)+\left[\left(\frac{b_{3}}{2 \delta k^{2}}\right)^{\frac{1}{4}}\left(u+\frac{b_{2}}{3 b_{3}}\right)-\gamma\right](\alpha-\beta)}{\left|\left[\left(\frac{b_{3}}{2 \delta k^{2}}\right)^{\frac{1}{4}}\left(u+\frac{b_{2}}{3 b_{3}}\right)-\alpha\right](\beta-\alpha)\right|} .
\end{aligned}
$$

If $\frac{b_{3}}{\delta}<0$, when $\alpha>\beta$ and $w>\beta$ or when $\alpha<\gamma$ and $w<\gamma$, we have

$$
\begin{aligned}
& \pm\left(\exp \left\{ \pm\left(-\frac{b_{3}}{2 \delta k^{2}}\right)^{\frac{1}{4}}(\alpha-\beta)(\alpha-\gamma)\left(\xi-\xi_{0}\right)\right\}\right)^{\frac{1}{2}} \\
& =\frac{\sqrt{\left[-\left(-\frac{b_{3}}{2 \delta k^{2}}\right)^{\frac{1}{4}}\left(u+\frac{b_{2}}{3 b_{3}}\right)-\beta\right](\gamma-\alpha)} \sqrt{\left[\left(-\frac{b_{3}}{2 \delta k^{2}}\right)^{\frac{1}{4}}\left(u+\frac{b_{2}}{3 b_{3}}\right)^{\frac{1}{4}}\left(u+\frac{b_{2}}{3 b_{3}}\right)-\gamma\right](\beta-\alpha)}}{\sqrt{\left|\left(-\frac{b_{3}}{2 \delta k^{2}}\right)^{\frac{1}{4}}\left(u+\frac{b_{2}}{3 b_{3}}\right)-\alpha\right|}},
\end{aligned}
$$


when $\alpha>\beta$ and $w<\gamma$, or when $\alpha<\gamma$, and $w<\beta$, we have

$$
\begin{aligned}
& \pm\left(\exp \left\{ \pm\left(-\frac{b_{3}}{2 \delta k^{2}}\right)^{\frac{1}{4}}(\alpha-\beta)(\alpha-\gamma)\left(\xi-\xi_{0}\right)\right\}\right)^{\frac{1}{2}} \\
& =\frac{\sqrt{\left[-\left(-\frac{b_{3}}{2 \delta k^{2}}\right)^{\frac{1}{4}}\left(u+\frac{b_{2}}{3 b_{3}}\right)-\beta\right](\alpha-\gamma)}}{\sqrt{\left|\left(-\frac{b_{3}}{2 \delta k^{2}}\right)^{\frac{1}{4}}\left(u+\frac{b_{2}}{3 b_{3}}\right)-\alpha\right|}}-\frac{\sqrt{\left[\left(-\frac{b_{3}}{2 \delta k^{2}}\right)^{\frac{1}{4}}\left(u+\frac{b_{2}}{3 b_{3}}\right)-\gamma\right](\alpha-\beta)}}{\sqrt{\left|\left(-\frac{b_{3}}{2 \delta k^{2}}\right)^{\frac{1}{4}}\left(u+\frac{b_{2}}{3 b_{3}}\right)-\alpha\right|}},
\end{aligned}
$$

when $\beta>\alpha>\gamma$, we have

$$
\begin{aligned}
& \pm \sin \left[\left(-\frac{b_{3}}{2 \delta k^{2}}\right)^{\frac{1}{4}}(\beta-\alpha)(\alpha-\gamma)\left(\xi-\xi_{0}\right)\right] \\
& =\frac{\left[-\left(-\frac{b_{3}}{2 \delta k^{2}}\right)^{\frac{1}{4}}\left(u+\frac{b_{2}}{3 b_{3}}\right)+\beta\right](\alpha-\gamma)+\left[\left(-\frac{b_{3}}{2 \delta k^{2}}\right)^{\frac{1}{4}}\left(u+\frac{b_{2}}{3 b_{3}}\right)-\gamma\right](\alpha-\beta)}{\left|\left[\left(-\frac{b_{3}}{2 \delta k^{2}}\right)^{\frac{1}{4}}\left(u+\frac{b_{2}}{3 b_{3}}\right)-\alpha\right](\beta-\alpha)\right|}
\end{aligned}
$$

where $\gamma=\frac{\alpha-2 l}{\sqrt{(\alpha-l)^{2}+s^{2}}}$, and $\delta=\sqrt{(\alpha-l)^{2}+s^{2}}-\frac{\alpha(\alpha-2 l)}{\sqrt{(\alpha-l)^{2}+s^{2}}}$.

Case 2.2.6. $D_{2}>0, D_{3}>0$, and $D_{4}>0, F(w)=\left(w-\alpha_{1}\right)\left(w-\alpha_{2}\right)\left(w-\alpha_{3}\right)\left(w-\alpha_{4}\right)$, where $\alpha_{1}>\alpha_{2}>\alpha_{3}>\alpha_{4}$. If $\frac{b_{3}}{\delta}>0$, when $w>\alpha_{1}$, or $w<\alpha_{4}$, the solution of Equation (7) is

$$
u=-\frac{b_{2}}{3 b_{3}}\left(\frac{b_{3}}{2 \delta k^{2}}\right)^{-\frac{1}{4}}\left[\frac{\alpha_{2}\left(\alpha_{1}-\alpha_{4}\right) \operatorname{sn}^{2}\left(\left(\frac{b_{3}}{2 \delta k^{2}}\right)^{\frac{1}{4}} \frac{\sqrt{\left(\alpha_{1}-\alpha_{3}\right)\left(\alpha_{2}-\alpha_{4}\right)}}{2}\left(\xi-\xi_{0}\right), m\right)-\alpha_{1}\left(\alpha_{2}-\alpha_{4}\right)}{\left(\alpha_{1}-\alpha_{4}\right) \operatorname{sn}^{2}\left(\left(\frac{b_{3}}{2 \delta k^{2}}\right)^{\frac{1}{4}} \frac{\sqrt{\left(\alpha_{1}-\alpha_{3}\right)\left(\alpha_{2}-\alpha_{4}\right)}}{2}\left(\xi-\xi_{0}\right), m\right)-\left(\alpha_{2}-\alpha_{4}\right)}\right] .
$$

when $\alpha_{3}<w<\alpha_{2}$, the solution of Equation (7) is

$$
u=-\frac{b_{2}}{3 b_{3}}\left(\frac{b_{3}}{2 \delta k^{2}}\right)^{-\frac{1}{4}}\left[\frac{\alpha_{4}\left(\alpha_{2}-\alpha_{3}\right) \operatorname{sn}^{2}\left(\left(\frac{b_{3}}{2 \delta k^{2}}\right)^{\frac{1}{4}} \frac{\sqrt{\left(\alpha_{1}-\alpha_{3}\right)\left(\alpha_{2}-\alpha_{4}\right)}}{2}\left(\xi-\xi_{0}\right), m\right)-\alpha_{3}\left(\alpha_{2}-\alpha_{4}\right)}{\left(\alpha_{2}-\alpha_{3}\right) \operatorname{sn}^{2}\left(\left(\frac{b_{3}}{2 \delta k^{2}}\right)^{\frac{1}{4}} \frac{\sqrt{\left(\alpha_{1}-\alpha_{3}\right)\left(\alpha_{2}-\alpha_{4}\right)}}{2}\left(\xi-\xi_{0}\right), m\right)-\left(\alpha_{2}-\alpha_{4}\right)}\right] .
$$

If $\frac{b_{3}}{2 \delta k^{2}}<0$, when $\alpha_{1}>w>\alpha_{2}$, the solution of Equation (7) is 


$$
u=-\frac{b_{2}}{3 b_{3}}\left(-\frac{b_{3}}{2 \delta k^{2}}\right)^{-\frac{1}{4}}\left[\frac{\alpha_{3}\left(\alpha_{1}-\alpha_{2}\right) \operatorname{sn}^{2}\left(\left(-\frac{b_{3}}{2 \delta k^{2}}\right)^{\frac{1}{4}} \frac{\sqrt{\left(\alpha_{1}-\alpha_{3}\right)\left(\alpha_{2}-\alpha_{4}\right)}}{2}\left(\xi-\xi_{0}\right), m\right)-\alpha_{2}\left(\alpha_{1}-\alpha_{3}\right)}{\left(\alpha_{1}-\alpha_{2}\right) \operatorname{sn}^{2}\left(\left(-\frac{b_{3}}{2 \delta k^{2}}\right)^{\frac{1}{4}} \frac{\sqrt{\left(\alpha_{1}-\alpha_{3}\right)\left(\alpha_{2}-\alpha_{4}\right)}}{2}\left(\xi-\xi_{0}\right), m\right)-\left(\alpha_{1}-\alpha_{3}\right)}\right],
$$

when $\alpha_{4}<w<\alpha_{3}$, the solution of Equation (7) is

$$
u=-\frac{b_{2}}{3 b_{3}}\left(-\frac{b_{3}}{2 \delta k^{2}}\right)^{-\frac{1}{4}}\left[\frac{\alpha_{1}\left(\alpha_{1}-\alpha_{2}\right) \operatorname{sn}^{2}\left(\left(-\frac{b_{3}}{2 \delta k^{2}}\right)^{\frac{1}{4}} \frac{\sqrt{\left(\alpha_{1}-\alpha_{3}\right)\left(\alpha_{2}-\alpha_{4}\right)}}{2}\left(\xi-\xi_{0}\right), m\right)-\alpha_{4}\left(\alpha_{3}-\alpha_{1}\right)}{\left(\alpha_{3}-\alpha_{4}\right) \operatorname{sn}^{2}\left(\left(-\frac{b_{3}}{2 \delta k^{2}}\right)^{\frac{1}{4}} \frac{\sqrt{\left(\alpha_{1}-\alpha_{3}\right)\left(\alpha_{2}-\alpha_{4}\right)}}{2}\left(\xi-\xi_{0}\right), m\right)-\left(\alpha_{3}-\alpha_{1}\right)}\right],
$$

where $m^{2}=\frac{\left(\alpha_{1}-\alpha_{2}\right)\left(\alpha_{3}-\alpha_{4}\right)}{\left(\alpha_{1}-\alpha_{3}\right)\left(\alpha_{2}-\xi_{4}\right)}$.

Case 2.2.7. $D_{2} D_{3} \geq 0$, and $D_{4}<0, F(w)=(w-\alpha)(w-\beta)\left((w-l)^{2}+s^{2}\right)$. where $\alpha>\beta$ and $s>0$.

The solution of Equation (7) (when $\frac{b_{3}}{\delta}>0$, we take the positive sign; when $\frac{b_{3}}{\delta}<0$, we take the negative) is

$$
u=\left( \pm \frac{b_{3}}{2 \delta k^{2}}\right)^{-\frac{1}{4}}\left[\frac{\left.\operatorname{acn}\left(\left( \pm \frac{b_{3}}{2 \delta k^{2}}\right)^{\frac{1}{4}} \frac{\sqrt{\mp 2 s m_{1}(\alpha-\beta)}}{2 m m_{1}}\left(\xi-\xi_{0}\right), m\right)+b\right]}{\operatorname{ccn}\left(\left( \pm \frac{b_{3}}{2 \delta k^{2}}\right)^{\frac{1}{4}} \frac{\sqrt{\mp 2 s m_{1}(\alpha-\beta)}}{2 m m_{1}}\left(\xi-\xi_{0}\right), m\right)+d}-\frac{b_{2}}{3 b_{3}}\right.
$$

where $a=\frac{1}{2}(\alpha+\beta) c-\frac{1}{2}(\alpha-\beta) d, \quad b=\frac{1}{2}(\alpha+\beta) d-\frac{1}{2}(\alpha-\beta) c, \quad c=\alpha-l-\frac{s}{m_{1}}, \quad d=\alpha-l-s m_{1}$, $E=\frac{s^{2}+((\alpha-l) \beta-l)}{s(\alpha-\beta)}, m_{1}=E \pm \sqrt{E^{2}+1}, m^{2}=\frac{1}{1+m_{1}^{2}}$.

Case 2.2.8. $D_{2} D_{3} \leq 0$, and $D_{4}>0$, then $F(w)=\left(\left(w-l_{1}\right)^{2}+s_{1}^{2}\right)\left(\left(w-l_{2}\right)^{2}+s_{2}^{2}\right)$, where $s_{1} \geq s_{2}>0$. The solution of Equation (7) is

$$
u=\left( \pm \frac{b_{3}}{2 \delta k^{2}}\right)^{-\frac{1}{4}}\left[\frac{a \operatorname{sn}\left(\eta\left(\xi_{1}-\xi_{0}\right), m_{1}\right)+b c n\left(\eta\left(\xi-\xi_{0}\right), m_{1}\right)}{\operatorname{csn}\left(\eta\left(\xi-\xi_{0}\right), m_{1}\right)+d c n\left(\eta\left(\xi_{1}-\xi_{0}\right), m_{1}\right)}\right]-\frac{b_{2}}{3 b_{3}}
$$

where $\eta=\frac{s_{2} \sqrt{\left(c^{2}+d^{2}\right)\left(m_{1}^{2} c^{2}+d^{2}\right)}}{c^{2}+d^{2}}, a=l_{1} c+s_{1} d, \quad b=l_{1} d-s_{1} c, \quad c=-s_{1}-\frac{s_{2}}{m_{1}}, \quad d=l_{1}-l_{2}$, $E=\frac{\left(l_{1}-l_{2}\right)^{2}+s_{1}^{2}+s_{2}^{2}}{2 s_{1} s_{2}}, m_{1}=E+\sqrt{E^{2}-1}$.

Case 2.2.9. $D_{2} D_{3}<0$ and $D_{4}=0$, then $F(w)=(w-\alpha)^{2}\left((w-l)^{2}+s\right)$, where $\alpha, l$ and $s$ are real numbers. If $\frac{b_{3}}{\delta}>0$, we have 


$$
u=\left(\frac{b_{3}}{2 \delta k^{2}}\right)^{-\frac{1}{4}}\left[\frac{\mathrm{e}^{ \pm\left(\frac{b_{3}}{2 \delta k^{2}}\right)^{-\frac{1}{4}} \sqrt{(l-l)^{2}+s^{2}}\left(\xi-\xi_{0}\right)}-\gamma+\sqrt{(\alpha-l)^{2}+s^{2}}(2-\gamma)}{\left[\mathrm{e}^{\left( \pm \frac{b_{3}}{2 \delta k^{2}}\right)^{-\frac{1}{4}} \sqrt{(l-l)^{2}+s^{2}}\left(\xi-\xi_{0}\right)}-\gamma\right]^{2}-1}\right]-\frac{b_{2}}{3 b_{3}},
$$

where $\gamma=\frac{\alpha-2 l}{\sqrt{(\alpha-l)^{2}+s^{2}}}$.

\section{Conclusion}

By the complete discrimination system for polynomial method, we have obtained the classification of single traveling wave solutions to the generalized strong nonlinear Boussinesq without dissipation terms in $p=1$.These solutions include trigonometric periodic solutions, rational function solution, hyperbolic funtion solutions, Jacobi elliptic function solutions and so on. This method is simple and efficient.

\section{Acknowledgements}

The project is supported by Scientific Research Fund of Education Department of Heilongjiang Province of China under Grant No. 12521049.

\section{REFERENCES}

[1] Fan, E.G. (1998) A Note on the Homogenous Balance Method. Physics Letters A, 246, 403-406. http://dx.doi.org/10.1016/S0375-9601(98)00547-7

[2] Ablowitz, M.J. and Clarkson, P.A. (1991) Solitons, Non-Linear Evolution Equations and Inverse Scattering Transform. Cambridge University Press, Cambridge. http://dx.doi.org/10.1017/CBO9780511623998

[3] Hirota, R. (1973) Exact Envelope-Soliton of a Nonlinear Wave Equation. Journal of Mathematical Physics, 14, 805-813. http://dx.doi.org/10.1063/1.1666399

[4] Ma, W.X. and Fuchssteiner, B. (1996) Explicit and Exact Solutions to a Kolmogorov-Petrovskii-Piskunov Equation. International Journal of Non-Linear Mechanics, 31, 329-338. http://dx.doi.org/10.1016/0020-7462(95)00064-X

[5] Ma, W.X. (1993) Travelling Wave Solutions to a Seventh Order Generalized KdV Equation. Physics Letters A, 180, $221-224$.

[6] Liu, C.S. (2010) Applications of Complete Discrimination System for Polynomial for Classifications of Traveling Wave Solutions to Nonlinear Differential Equations. Computer Physics Communications, 181, 317-324. http://dx.doi.org/10.1016/j.cpc.2009.10.006

[7] Liu, C.S. (2007) Classification of All Single Traveling Wave Solutions to Calogero-Focas Equation. Communications in Theoretical Physics (Beijing), 48, 601-604. http://dx.doi.org/10.1088/0253-6102/48/4/004

[8] Liu, C.S. (2006) Direct integral method, complete discrimination system for polynomial and applications to classifications of all single travelling wave solutions to nonlinear differential equations: a survey. arXiv: nlin/0609058v1

[9] Zhang, W.G. and Tao, T. (2008) Analysis of Solitary-Wave Shape and Solutions of the Generalized Strong Nonlinear Boussinesq Equation. Acta Mathematica Sientia, 28A, 086-095.

[10] Whitham, G.B. (1974) Linear and Nonlinear Wave. Springer, New York.

[11] Zhakarov, V.E. (1974) On Stochastization of One-Dimensional Chains of Nonlinear Oscillation. Soviet Physics-JETP, 38, 108110.

[12] McKean, H.P. (1981) Boussinesq’s Equation on the Circle. Pure and Applied Mathematics, 34, 599-690. http://dx.doi.org/10.1002/cpa.3160340502

[13] Manoranjan, V.S., et al. (1985) Numerical Solution of the Good Boussinesq Equation. SIAM: SIAM Journal on Scientific Computing, 5, 946-957.

[14] Weiss, J. (1985) The Painlevé Property and Backlund Transformation for the Sequence of Boussinesq Equations. Journal of Mathematical Physics, 26, 258-269. http://dx.doi.org/10.1063/1.526655

[15] Hu, X.G., Wu, Y.H. and Li, L. (2013) New Traveling Wave Solutions of the Boussinesq Equation Using a New Generalized 
Mapping Method. Journal of Basic and Applied Physics, 2, 68-77. http://dx.doi.org/10.5963/JBAP0202005

[16] Zakharov, V.E., et al. (1984) Theory of Solitons: The Iverse Scattering Method. Plenum Press, New York.

[17] Hirota, R. (1973) Exact N-Soliton Solutions of the Wave Equation of Long Wave Shallow-Water and in Nonlinear Lattices. Journal of Mathematical Physics, 14, 810-814. http://dx.doi.org/10.1063/1.1666400 